\title{
On the Dimension Formula for the Hyperfunction Solutions of Some Holonomic D-modules
}

\author{
By \\ Jörg SChÜRMANN*
}

\begin{abstract}
In this short note we improve a recent dimension formula of Takeuchi for the dimension of the hyperfunction solutions of some holonomic D-modules. Besides the constructibility result and the local index formula of Kashiwara for the holomorphic solution complex, we only use a vanishing theorem of Lebeau together with a simple calculation in terms of constructible functions.
\end{abstract}

\section{$\S 1$. Introduction}

One of the basic results about holonomic D-modules is the constructibility result of Kashiwara [6], that the holomorphic solution complex

$$
\operatorname{Sol}(\mathcal{M}):=\operatorname{Rhom}_{D_{X}}\left(\mathcal{M}, \mathcal{O}_{X}\right)
$$

of a holonomic D-module $\mathcal{M}$ on the complex manifold $X$ is a bounded complex analytically constructible complex of sheaves of complex vector spaces with finite dimensional stalks (compare also with [7, chapter 5], [10, thm.4.5.8, p.458] and $[14$, chap.III]). In particular, the function

$$
\chi(\mathcal{M}): X \rightarrow \mathbb{Z} ; x \mapsto \chi\left(\operatorname{Sol}(\mathcal{M})_{x}\right)
$$

is well defined and complex analytically constructible. Here $\chi$ is the usual Euler characteristic. Moreover, one has by Kashiwara [5] the following beautiful

Communicated by M. Kashiwara. Received July 15, 2003.

2000 Mathematics Subject Classification(s): 32C38, 32S40, 35A27.

*Westf. Wilhelms-Universität, SFB 478 "Geometrische Strukturen in der Mathematik", Hittorfstr.27, 48149 Münster, Germany.

e-mail: jschuerm@math.uni-muenster.de

(c) 2006 Research Institute for Mathematical Sciences, Kyoto University. All rights reserved. 
description of this local index in terms of the characteristic cycle of the holonomic D-module $\mathcal{M}$ (see [7, thm.6.3.1, p.127, cor.6.3.4, p.128] and [2, thm.2, p.574]):

Theorem 1.1 (local index formula). Let $Y_{j}$ be finitely many distinct irreducible closed complex analytic subsets of $X$ such that the characteristic variety $\operatorname{char}(\mathcal{M})$ of $\mathcal{M}$ can be estimated by

$$
\operatorname{char}(\mathcal{M}) \subset \bigcup_{j} T_{Y_{j}}^{*} X, \quad \text { with } \quad T_{Y}^{*} X:=\operatorname{cl}\left(T_{Y_{\text {reg }}^{*}}^{*} X\right)
$$

the closure of the conormal bundle to the regular part $Y_{\text {reg }}$ of the irreducible complex analytic subset $Y \subset X$. Then

$$
\chi(\mathcal{M})(x)=\sum_{j}(-1)^{d_{j}} \cdot m_{j} \cdot E u_{Y_{j}}(x),
$$

with $d_{j}$ the complex codimension of $Y_{j}, E u_{Y_{j}}$ the famous Euler obstruction of $Y_{j}$ as defined by MacPherson (cf. [13]) and $m_{j}$ the (generic) multiplicity of $\mathcal{M}$ along $T_{Y_{j}}^{*} X$.

Note that $E u_{Y}=1_{Y}$, if $Y \subset X$ is a closed complex analytic submanifold. So a very special case of the local index formula is given as in [7, ex. on p.129] by the

Example 1. Suppose all $Y_{j}$ in the estimate (2) are closed connected complex analytic submanifolds of $X$. Then

$$
\chi(\mathcal{M})(x)=\sum_{j}(-1)^{d_{j}} \cdot m_{j} \cdot 1_{Y_{j}}(x),
$$

with $d_{j}$ the complex codimension of $Y_{j}$ and $m_{j}$ the (generic) multiplicity of $\mathcal{M}$ along $T_{Y_{j}}^{*} X$.

Let us now consider the case that $X$ is the complexification of the real analytic manifold $M$, with $i: M \hookrightarrow X$ the closed inclusion. Assume $M$ is purely n-dimensional. Then the sheaf complex

$$
R \Gamma_{M}\left(\mathcal{O}_{X}\right)[n] \simeq R i_{*} i^{!}\left(\mathcal{O}_{X}\right)[n]
$$

is concentrated in degree zero, with

$$
\mathcal{B}_{M}:=h^{0}\left(i^{!}\left(\mathcal{O}_{X}\right)[n]\right) \otimes o r_{M}
$$

the sheaf of Sato's hyperfunctions on $M$, and or $_{M}$ the orientation sheaf of $M$. 
Then the hyperfunction solution complex

$$
\operatorname{Rhom}_{i^{*} D_{X}}\left(i^{*} \mathcal{M}, \mathcal{B}_{M}\right) \simeq i^{!} \operatorname{Sol}(\mathcal{M}) \otimes \operatorname{or}_{M}[n]
$$

of a holonomic D-module $\mathcal{M}$ on $X$ is subanalytically constructible on $M$ with finite dimensional stalks (compare [7, thm.5.1.7, p.115]). So it is natural to ask for a corresponding index formula like (3) or (4).

Remark. The same constructibility result is true for the solutions

$$
\operatorname{Rhom}_{i^{*} D_{X}}\left(i^{*} \mathcal{M}, \mathcal{A}_{M}\right) \simeq i^{*} \operatorname{Sol}(\mathcal{M})
$$

in the sheaf $\mathcal{A}_{M}=i^{*} \mathcal{O}_{X}$ of real analytic functions on $M$. If $\mathcal{M}$ is a regular holonomic D-module on $X$, then one also has isomorphisms (see [8, cor.8.3, cor.8.5, p.360] or $[1, \mathrm{p} .326])$ :

$$
\operatorname{Rhom}_{i^{*} D_{X}}\left(i^{*} \mathcal{M}, \mathcal{B}_{M}\right) \simeq \operatorname{Rhom}_{i^{*} D_{X}}\left(i^{*} \mathcal{M}, \mathcal{D} b_{M}\right)
$$

and

$$
\operatorname{Rhom}_{i^{*} D_{X}}\left(i^{*} \mathcal{M}, \mathcal{A}_{M}\right) \simeq \operatorname{Rhom}_{i^{*} D_{X}}\left(i^{*} \mathcal{M}, \mathcal{C}_{M}^{\infty}\right),
$$

with $\mathcal{D} b_{M}$ (or $\mathcal{C}_{M}^{\infty}$ ) the sheaf of distributions (or smooth functions) on $M$.

The following counterpart of (4) is the main result of this note:

Theorem 1.2 (local dimension formula). Let $M_{j}$ be finitely many distinct closed real analytic submanifolds of $M$ such that the characteristic variety char $(\mathcal{M})$ of the holonomic $D$-module $\mathcal{M}$ on $X$ can be estimated by

$$
\operatorname{char}(\mathcal{M}) \subset \bigcup_{j} T_{Y_{j}}^{*} X
$$

with $Y_{j} \subset X$ the complexification of $M_{j}$. Assume the $Y_{j}$ are irreducible (i.e. connected), with $Y_{j} \cap M=M_{j}$. Then one has for $x \in M$ :

$$
\operatorname{dim}_{C}\left(\operatorname{hom}_{i^{*} D_{X}}\left(i^{*} \mathcal{M}, \mathcal{B}_{M}\right)_{x}\right)=\sum_{j} m_{j} \cdot 1_{M_{j}}(x),
$$

with $m_{j}$ the (generic) multiplicity of $\mathcal{M}$ along $T_{Y_{j}}^{*} X$.

This is indeed a counterpart of (4). The estimate (6) implies by a theorem of Lebeau [12] (compare also with [3, thm.2.1, rem., p.531] and [3, ex.(1), p.533]) the vanishing result

$$
\mathcal{E} x t_{i^{*} D_{X}}^{k}\left(i^{*} \mathcal{M}, \mathcal{B}_{M}\right)_{x}=0 \quad \text { for all } k \geq 1
$$


so that

$$
\operatorname{dim}_{C}\left(\operatorname{hom}_{i^{*} D_{X}}\left(i^{*} \mathcal{M}, \mathcal{B}_{M}\right)_{x}\right)=\chi\left(\operatorname{Rhom}_{i^{*} D_{X}}\left(i^{*} \mathcal{M}, \mathcal{B}_{M}\right)_{x}\right) .
$$

Theorem 1.2 answers affirmatively a question asked (or better, discussed) in [17, rem.3.5] at the end of a recent paper of Takeuchi [17], where he proves the dimension formula (7) under the special assumption, that in suitable local coordinates $(M, x) \simeq\left(\mathbb{R}^{n}, 0\right)$ the $M_{j}$ are linear subspaces (passing through $x=0)$.

Note that this special case already covers (locally) the one-dimensional case $(X, M, x) \simeq(\mathbb{C}, \mathbb{R}, 0)$, with $\mathcal{M}$ a holonomic D-module such that

$$
\operatorname{char}(\mathcal{M}) \subset T_{\{x\}}^{*} X \cup T_{X}^{*} X .
$$

In this case one gets back a classical result of Kashiwara [4, thm.4.2.7, p.69] (cf. [7, cor.3.2.36(b), p.88-89]) and Komatsu [11]:

$$
\operatorname{dim}_{C}\left(\operatorname{hom}_{i^{*} D_{X}}\left(i^{*} \mathcal{M}, \mathcal{B}_{M}\right)_{x}\right)=d+d^{\prime},
$$

with $d$ or $d^{\prime}$ the multiplicity of $\mathcal{M}$ along $T_{\{x\}}^{*} X$ or $T_{X}^{*} X$.

Let $j: X \backslash M \rightarrow M$ be the open inclusion of the complement of $M$ in $X$. Then the proof given in [17, sec.3] is based on the distinguished triangle

$$
R i_{*} i^{\prime} \operatorname{Sol}(\mathcal{M})[n] \longrightarrow \operatorname{Sol}(\mathcal{M})[n] \longrightarrow R j_{*} j^{*} \operatorname{Sol}(\mathcal{M})[n] \stackrel{[1]}{\longrightarrow}
$$

Moreover, he uses the micro-local theory of the characteristic cycles $C C(\cdot)$ for subanalytically constructible complexes of sheaves (as in $[9,10,15,16]$ ), in particular a deep result of Schmid-Vilonen [15] about a description of

$$
C C\left(R j_{*} j^{*} \operatorname{Sol}(\mathcal{M})\right) \text { in terms of } C C(\operatorname{Sol}(\mathcal{M})) .
$$

In the next section we explain our simple proof of Theorem 1.2, which doesn't make use of this sophisticated micro-local theory of characteristic cycles. Instead of this, we use the observation that the calculation of

$$
\chi\left(\left(i^{!} \mathcal{F}\right)_{x}\right) \quad \text { for } \quad \mathcal{F}=\operatorname{Sol}(\mathcal{M})
$$

can be done in terms of subanalytically constructible functions, i.e. the functor $i^{!}$induces a corresponding (unique) $\mathbb{Z}$-linear transformation for the abelian groups $C F(\cdot)$ of subanalytically constructible functions such that the following 
diagram commutes (compare [10, sec.9.7] and [16, sec.2.3]):

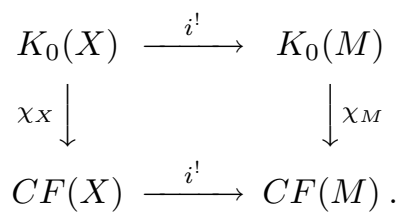

Here $K_{0}(\cdot)$ is the Grothendieck group of subanalytically constructible (complexes of) sheaves with finite dimensional stalks, with $\chi$ ? induced by taking stalkwise the Euler characteristic.

Then the calculation of

$$
\chi\left(\left(i ! \operatorname{Sol}(\mathcal{M}) \otimes \operatorname{or}_{M}[n]\right)_{x}\right)=(-1)^{n} \cdot i^{!}\left(\chi_{X}(\mathcal{S o l}(\mathcal{M}))\right)(x)
$$

becomes an easy exercise by the local index theorem and example 1 , since the $M_{j}$ and therefore also the $Y_{j}$ are closed submanifolds!

If we allow in the estimate (6) also singular subspaces, then we get at least the following weak parity version of the local index theorem:

Theorem 1.3 (local index formula for hyperfunctions). Let $M_{j}$ be finitely many distinct real analytic subspaces of $M$ such that the characteristic variety char $(\mathcal{M})$ of the holonomic $D$-module $\mathcal{M}$ on $X$ can be estimated as in (6), with $Y_{j} \subset X$ the complexification of $M_{j}$. Assume the $Y_{j}$ are irreducible, with $Y_{j} \cap M=M_{j}$. Then one has for $x \in M$ :

$$
\chi\left(\operatorname{Rhom}_{i^{*} D_{X}}\left(i^{*} \mathcal{M}, \mathcal{B}_{M}\right)_{x}\right) \equiv \sum_{j} m_{j} \cdot E u_{Y_{j}}(x) \quad \bmod 2,
$$

with $E u_{Y_{j}}$ the Euler obstruction of $Y_{j}$ and $m_{j}$ the (generic) multiplicity of $\mathcal{M}$ along $T_{Y_{j}}^{*} X$.

\section{$\S 2$. Constructible Functions}

In this final section we give the proof of Theorem 1.2 and 1.3 in terms of constructible functions. Let us start with the proof of Theorem 1.2.

By the estimate (6) and Example 1 we get

$$
\chi_{X}(\operatorname{Sol}(\mathcal{M}))=\sum_{j}(-1)^{d_{j}} \cdot m_{j} \cdot 1_{Y_{j}},
$$


with $d_{j}$ the complex codimension of $Y_{j}$ and $m_{j}$ the (generic) multiplicity of $\mathcal{M}$ along $T_{Y_{j}}^{*} X$. By linearity of $i^{!}$on the level of constructible functions one also has

$$
i^{!}\left(\chi_{X}(\mathcal{S} o l(\mathcal{M}))\right)=\sum_{j}(-1)^{d_{j}} \cdot m_{j} \cdot i^{!}\left(1_{Y_{j}}\right) .
$$

Then the dimension formula (7) follows from (5), (9), (12) and the simple formula

$$
i^{!}\left(1_{Y_{j}}\right)=(-1)^{\left(n-d_{j}\right)} \cdot 1_{M_{j}} .
$$

The formula (14) corresponds by the commutative diagram (11) to the base change formula

$$
i^{!}\left(R k_{*} \mathbb{C}_{Y_{j}}\right) \simeq R k_{*}^{\prime} i^{\prime !} \mathbb{C}_{Y_{j}}
$$

for the cartesian diagram of inclusions

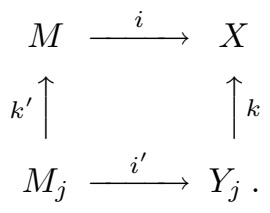

Note that $i^{\prime !} \mathbb{C}_{Y_{j}} \simeq \mathbb{C}_{M_{j}}\left[-\left(n-d_{j}\right)\right]$ locally on $M_{j}$, since $M_{j}$ is a closed submanifold of $Y_{j}$ of real codimension equal to the complex dimension $n-d_{j}$ of $Y_{j}$.

For the proof of the parity formula (13) in Theorem 1.3 it is enough to show

$$
\chi\left(i^{*} R j_{*} j^{*} \operatorname{Sol}(\mathcal{M})_{x}\right) \equiv 0 \quad \bmod 2 \text { for all } x \in M .
$$

Use the local index formula (3) and the distinguished triangle (10). But this follows from the fact that the constructible function

$$
\chi_{X}(\mathcal{S o l}(\mathcal{M})) \bmod 2
$$

is invariant under the complex conjugation acting on the complexification $X$ of $M$ (with fixed point set $M$ ).

More precisely, by [16, lem.1.1.1, p.27] one gets the description:

$$
\chi\left(i^{*} R j_{*} j^{*} \operatorname{Sol}(\mathcal{M})_{x}\right)=\chi\left(R \Gamma\left(M_{f, x}, \mathcal{S o l}(\mathcal{M})\right)\right),
$$

with

$$
M_{f, x}:=\{\|x\| \leq \delta, f=w\} \quad \text { for } \quad 0<w \ll \delta \ll 1
$$


(i.e. for $w, \delta$ small, with $w$ also small compared to $\delta$ ) a local right Milnor fiber of the function $f$ at $x$, defined in local coordinates

$$
(X, M, x) \simeq\left(\mathbb{C}^{n}, \mathbb{R}^{n}, 0\right) \quad \text { by } \quad z=\left(z_{1}, \ldots, z_{n}\right) \mapsto f(z):=\sum_{k=1}^{n} i m\left(z_{k}\right)^{2} .
$$

Here $i m(\cdot)$ is the imaginary part, with the complex conjugation acting on $\left(\mathbb{C}^{n}, \mathbb{R}^{n}, 0\right)$ in the usual way. This conjugation leaves the compact semi-analytic set $M_{f, x}$ invariant without any fixed point! But the Euler characteristic

$$
\chi\left(R \Gamma\left(M_{f, x}, \mathcal{S} o l(\mathcal{M})\right)\right) \bmod 2
$$

can be calculated in terms of $\mathbb{Z}_{2}$-valued constructible functions:

$$
\chi\left(R \Gamma\left(M_{f, x}, \mathcal{S} \circ \mathrm{l}(\mathcal{M})\right)\right) \equiv(c \circ \pi)_{*} \alpha \bmod 2,
$$

with

$$
\begin{gathered}
\alpha:=\chi_{M_{f, x}}\left(\operatorname{Sol}(\mathcal{M}) \mid M_{f, x}\right) \bmod 2 \in C F\left(M_{f, x}, \mathbb{Z}_{2}\right), \\
\pi: M_{f, x} \rightarrow M_{f, x} / \operatorname{conj} . \quad \text { the quotient and } \quad c: M_{f, x} / \text { conj. } \rightarrow\{p t\}
\end{gathered}
$$

a constant map. Here $(c \circ \pi)_{*}$ is induced by $R(c \circ \pi)_{*}$ similarly as in (11) by the commutative diagram (compare $[10$, sec.9.7] and $[16$, sec.2.3]):

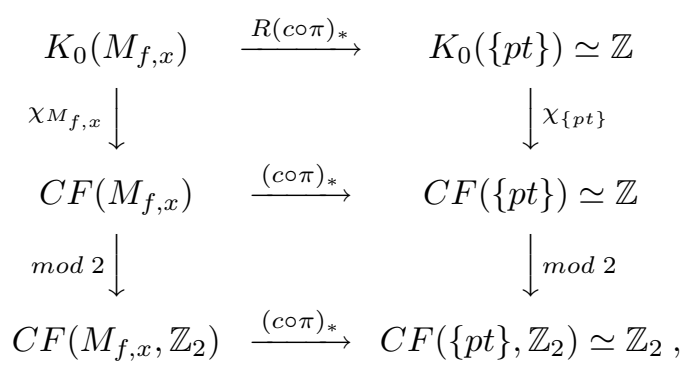

with $C F\left(\cdot, \mathbb{Z}_{2}\right)$ the corresponding abelian group of $\mathbb{Z}_{2}$-valued subanalytically constructible functions.

Then $(c \circ \pi)_{*}=c_{*} \circ \pi_{*}$ by functoriality. But $\pi_{*}(\alpha) \equiv 0$, since $\alpha$ is invariant under the conjugation conj., with $\pi: M_{f, x} \rightarrow M_{f, x} / c o n j$. an unramified covering of degree two. Of course, here it is important to work with $\mathbb{Z}_{2}$-valued constructible functions. 


\section{References}

[1] Björk, J. E., Analytic D-modules and applications, Kluwer, 1993.

[2] Brylinski, J. L., Dubson, A. and Kashiwara, M., Formule d'indice pour les modules holonomes et obstruction d'Euler locale, C. R. Acad. Sci. Paris, 293 (1981), 573-576.

[3] Honda, N. and Schapira, P., A vanishing theorem for holonomic modules with positive characteristic varieties, Publ. RIMS, Kyoto Univ., 26 (1990), 529-534.

[4] Kashiwara, M., Algebraic study of systems of linear differential equations, Master's Thesis, University of Tokyo 1970, English translation in: Mémoires de la SMF, 63, 1996.

[5] Index theorem for maximally overdetermined systems of linear differential equations, Proc. Japan Acad., 49 (1973), 803-804.

[6] , On the maximally overdetermined systems of linear differential equations I, Publ. RIMS, Kyoto Univ., 10 (1975), 563-579.

[7] _ Systems of microdifferential equations, Progr. Math., 34, Birkhäuser, Basel, 1983.

[8] The Riemann-Hilbert problem for holonomic systems, Publ. RIMS, Kyoto Univ., 20 (1984), 319-365.

[9] Index theorem for constructible sheaves, Astérisque, 130 (1985), 193-209.

[10] Kashiwara, M. and Schapira, P., Sheaves on Manifolds, Springer, Berlin Heidelberg, 1990.

[11] Komatsu, H., On the index of ordinary differential operators, J. Fac. Sci. Univ. Tokyo Sect. IA Math., 18 (1971), 379-398.

[12] Lebeau, G., Annulation de la cohomologie hyperfonction de certains modules holonomes, C. R. Acad. Sci. Paris Ser. A-B, 290 (1980), A313-A316.

[13] MacPherson, R., Chern classes for singular varieties, Ann. of Math., 100 (1974), 423432.

[14] Mebkhout, Z. and Narnáez-Macarro, L., Le Théorème de Constructibilité de Kashiwara, In: Images directes et constructibilité, ed. Maisonobe, M. and Sabbah, C., Travaux en cours, Hermann, Paris, 46 (1997), 47-98.

[15] Schmid, W. and Vilonen, K., Characteristic cycles for constructible sheaves, Inv. Math., 124 (1996), 451-502.

[16] Schürmann, J., Topology of singular spaces and constructible sheaves, Monografie Matematyczne (New Series), 63, Birkhäuser, Basel, 2003.

[17] Takeuchi, K., Dimension formulas for the hyperfunction solutions to holonomic D-modules, Adv. Math., 180 (2003), 134-145. 